\title{
Management of Severe Colitis in the Setting of Experimental Immunotherapy Combination
}

\author{
Alejandro Garcia-Alvarez ${ }^{1}$, Alberto Hernando ${ }^{1}$, Fabiola Amair-Pinedo ${ }^{1}$ and Eva Muñoz Couselo ${ }^{1,2}$ \\ ${ }^{1}$ Department of Medical Oncology, Vall d'Hebron University Hospital, Spain \\ ${ }^{2}$ Vall d'Hebron Institute of Oncology (VIHO), Spain
}

Submission: January 02, 2018; Published: April 18, 2018

*Corresponding author: Alejandro Garcia-Alvarez, Department of Medical Oncology, Vall d'Hebron University Hospital, 08035 Barcelona, Spain, Tel: +34 9348930 00; Email: alejandro.garcia@vhebron.net

Abstract

Introduction: Immune checkpoint inhibitors have changed the landscape for the treatment of metastatic melanoma and other solid malignancies. With their increasing use, physicians are facing a new spectrum of immune-related adverse effects. Diarrhea and enterocolitis are among the most common adverse effects.

Case report: We report the case of a patient with advanced metastatic malignant melanoma, previously treated with anti- Cytotoxic T-Lymphocyte Antigen 4 (anti-CTLA4) and anti- Programmed Death-1 (anti-PD-1) antibodies, who developed grade 3 immune-related colitis under experimental treatment with anti-PD-1 and anti-Lymphocyte Activation Gene 3 (anti-LAG3) antibodies. She received treatment with highdose intravenous glucocorticosteroids with favorable evolution.

Discussion: Treatment of anti-PD-1 and anti-CTLA4 mediated grade 2-3 colitis consists in intravenous glucocorticosteroids. However, no treatment has been previously described for colitis related to anti-LAG3 treatment. The case of our patient highlights the fact that corticosteroids are nowadays the mainstream treatment for any immunotherapy related colitis and is an evidence of efficacy in the context of anti-LAG3 treatment.

Keywords: Melanoma; Colitis; anti-LAG3; Checkpoint inhibitors; Immune-related colitis

\section{Introduction}

Treatment of metastatic melanoma has profoundly changed in the last years. The durable and even complete responses achieved with immunotherapy have changed the therapeutical objectives from quality of life maintenance and symptoms management to increase in overall survival [1].

Thus, based on the results of several phase III trials which included more than 4400 patients [1-4], two therapeutic strategies have been approved for first-line treatment in unresectable or metastatic melanoma, namely: antibodies against the immunecheckpoint modulators Cytotoxic T-Lymphocyte Antigen 4 (CTLA4) and Programmed Death-1 (PD-1) receptors, either alone or combined [1-3], and molecules that inhibit products of the oncogenes BRAF and MEK in those tumors which carry mutations in the BRAF gene [4]. The checkpoint inhibitors Ipilimumab (antiCTLA4 antibody), Nivolumab and Pembrolizumab (anti-PD-1) enhance the host antitumor immunity via T-cell activation and proliferation [5]. Although $20 \%$ of patients under Ipilimumab [1] and $34 \%$ of patients under Nivolumab [6] maintain response at 10 and 5 years respectively, early disease progression has been noted with immunotherapy agents. This fact compels the search of new targets that might achieve increased and maintained responses.
The Lymphocyte Activation Gene 3 (LAG3) is a membrane protein upregulated in activated $\mathrm{T}$ cells that binds the Major Histocompatibility Complex (MHC) Class II molecules with high affinity [7]. Similarly to PD-1 and CTLA-4, LAG3 negatively regulates $\mathrm{T}$ cells and is over expressed in tumor infiltrating lymphocytes (TILs) which suggest that this membrane protein might be a feasible target for tumoral immune modulation $[7,8]$.

Several trials demonstrate that immunotherapy is well tolerated and adverse effects associated with these treatments are mostly grade 1 or 2 (G1-G2) according to the Common Terminology Criteria for Adverse Events (CTCAE) Version 4.0 [5]. Nevertheless, the evidence of a wider spectrum of adverse effects (AE) is growing as their use increase. Undesired adverse effects are thought to be related to the systemic activation of the immune system [5].

As new immunotherapy drugs and combinations develop physicians are constantly required to learn related adverse events, toxicities and their management with a multidisciplinar approach. This will allow creating and improving work-up algorithms. In this scenario, education of patients on early recognition of adverse events related with immunotherapy is essential. Here, we report the case of a patient with advanced metastatic malignant melanoma 
treated with anti-CTL4, anti-PD-1 and anti-LAG3 antibodies who developed G3 immune-related colitis.

\section{Case Report}

A 67-year-old woman with no previous medical history was diagnosed in July 2014 of malignant nodular melanoma on her right leg. The main lesion was a $1.5 \mathrm{~cm}$ diameter papule surrounded by two smaller satellite lesions (less than $1 \mathrm{~cm}$ of diameter). Punch biopsy histology showed signs compatible with malignant nodular melanoma, Breslow's depth 2,8mm, no vascular invasion neither tumoral necrosis. Baseline LDH levels were 465UI/L. The tumour was BRAF wild type. Selective sentinel lymph node biopsy discarded local lymphatic invasion and PET-TC showed no distant metastases. The tumor stage corresponded to T2 N0 M1a (Stage IV).

First-line metastatic treatment based on Dacarbazine (DTIC, $1000 \mathrm{mg} / \mathrm{m} 2$ every 3 weeks) was administered in November 2014. After 2 cycles of treatment, local cutaneous progression was observed. In December 2014, Ipilimumab (3mg/kg every 3 weeks) was initiated as second-line treatment. The patient received four cycles showing Stable Disease (SD) as best response. A delay of the dose between cycle 3 and 4 was required due to Grade 2 enteritis. In May 2015, after six months of therapy, a CT scan showed nodal progression.

The patient was recruited into a phase III clinical trial for patients with unresectable stage III or stage IV melanoma progressing to an anti-CTLA4 monoclonal antibody, receiving Nivolumab since July 2015. Partial Response (PR) was assessed after 3 cycles. Local progression was observed by CT scan after 11 months and 21 cycles of Nivolumab. G1 asthenia was the only adverse effect reported within the time of treatment.
After progression, in July 2016, the patient was included in the phase I/IIa clinical trial (Clinicaltrials.gov NCT 01968109) and randomized to receive the combination of Nivolumab $(3 \mathrm{mg} / \mathrm{kg}$ every week) and anti-LAG3 (1mg/kg every week).

At week 8, treatment was stopped because of G3 diarrhea (5 to 7 non-bloody stools per day). The patient was admitted the on July 2016 in the Emergency Room. When admitted the patient was febrile, without tachycardia or hypotension. Her abdomen was benign, with no tenderness or distension and normal bowel sounds. White blood cell count was normal. Abdominal X-Ray showed colonic haustra without signs of bowel inflammation. Infectious colitis was ruled out by negative bacteriological, virological and parasitological analyses of stools including Clostridium difficile toxin. Blood cultures were also negative.

Under suspicion on diagnosis of immune-mediated colitis in a patient under experimental immunotherapy in which infectious etiology has been ruled out, methylprednisolone $1 \mathrm{mg} / \mathrm{Kg} /$ day was started, according to established management guidelines [5].

After 3 days of treatment, diarrhea worsened to 7-8 bloody stools per day. Taking into account the initial presumption diagnosis and the complementary tests performed so far, an endoscopic exploration was requested. Colonoscopy showed erythema, loss of vascular pattern and small ulcers (up to $7 \mathrm{~mm}$ of diameter) all along the colonic mucosa from the rectum to the hepatic flexure. Biopsies showed ulcerated mucosa with polymorphonuclear infiltrate of the lamina propia as well as cryptitis and crypt abscesses (Figure $1 \& 2$ ). These findings have been previously described in immunotherapyinduced colitis [9]. Methylprednisolone dose was then increased to $2 \mathrm{mg} / \mathrm{Kg} /$ day.

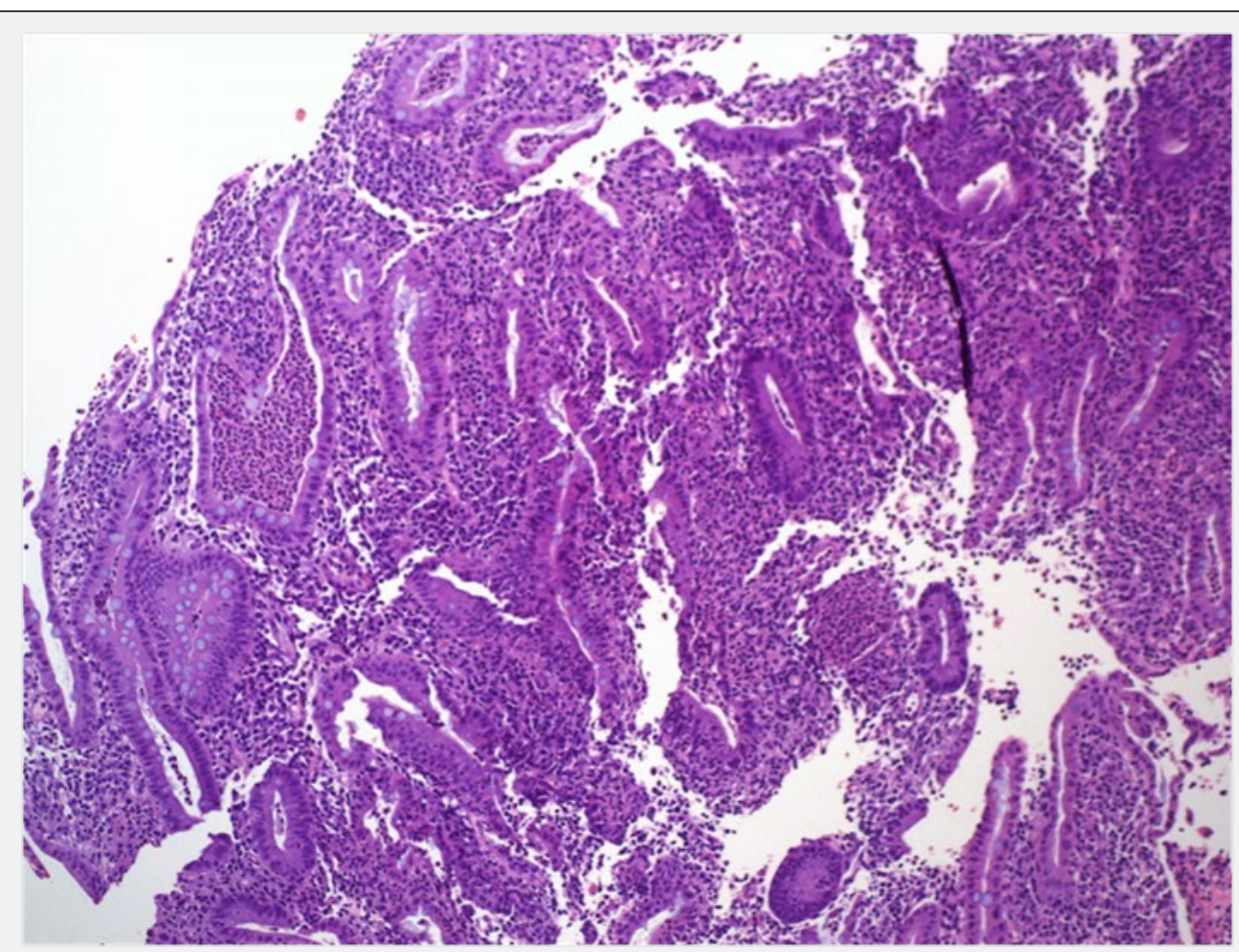

Figure 1: Colonic mucosa showing polymorphonuclear infiltrate (magnifying lens $\times 10$ ). Hematoxylin and eosin stain. 


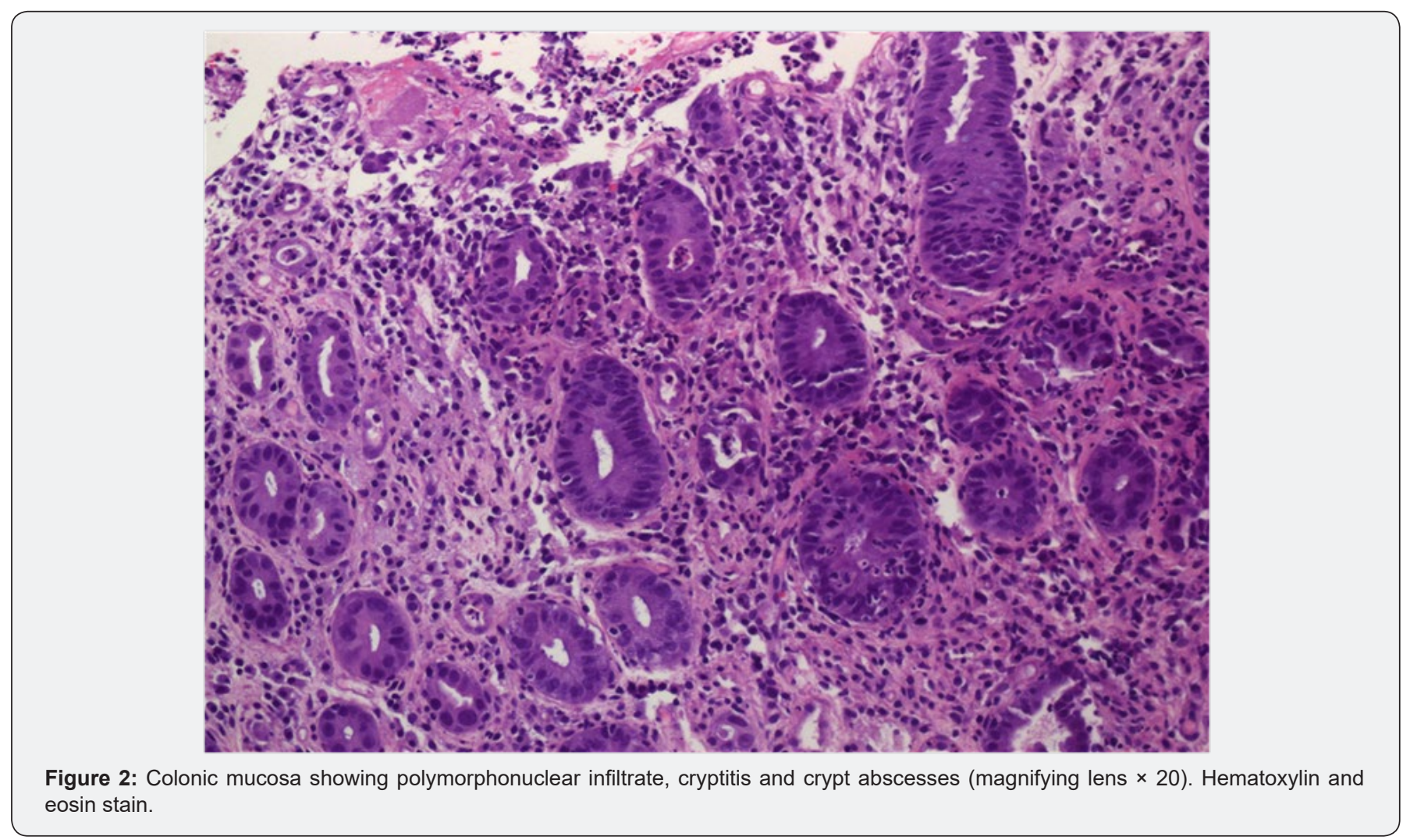

The patient symptoms improved in the following 72 hours. The number of stools decreased progressively over 10 days and the patient initiated oral intake. After 21 days of intravenous methylprednisolone, oral prednisone (at equivalent dosage) was started. The patient continued with 1-2 stools per day with oral corticoids tapered off along 50 days after discharge.

\section{Discussion}

Immune checkpoint inhibitors have changed the landscape for the treatment of metastatic melanoma and other solid malignancies [1-3]. With the increasing use of immune-based therapies for oncology patients, physicians are likely to see a growing burden of immune-mediated gastrointestinal toxicities [10]. Similarly to Anti PD1/PDL1, anti-LAG 3 modifies self-regulation of immune system, increasing the host anti-tumor response. Hence, this might increase the incidence of immune adverse effects [11].

Diarrhea and enterocolitis are among the most common immune-related AE. Gastrointestinal symptoms usually appear within 6-8 weeks after initiating the immune checkpoint regulators therapy [5]. Incidences of G3-4 enterocolitis vary between 1-3\% in anti-PD-1 therapy, being less frequent in patients treated with antiCTLA4 [3,5]. However, It has been reported up to $8 \%$ of G3-4 colitis in patients treated with combined checkpoint inhibitors [3], and the incidence of these events after concomitantly receiving anti-PD-1 and anti-LAG3 antibodies was one of the endpoints of the trial in which was the patient enrolled when the gastrointestinal event was reported.

Up to date there is no evidence that previous treatment with anti-CTLA4 increases the toxicity of subsequent anti-PD-1 treatments. However, little is known about the safety profile of patients treated with anti-PD-1 and anti-LAG 3 combination.

Immune mediated-colitis as usually reveals erythema, mucosal friability and ulceration, predominantly in distant colon [9]. Even though colonic ulceration has been described as an independent predictor of steroid-refractory course in enterocolitis related to anti-CTLA4 [12], our patient did show a favorable evolution as she improved in less than 48 hours after corticosteroids dose increment.

Pathologic features described in anti-CTLA4 related colitis include both neutrophilic and lymphocytic infiltrates which are frequently observed in chronic inflammatory bowel disease [9]. No biopsy proven colitis with anti-PD-1/PD-L1 has been reported to date and in our case, other potential entities as infectious colitis were ruled out and the patient lacked of a history of autoimmune disorders. Hence, the changes observed in the colon mucosa may be attributed to the experimental treatment.

Treatment of anti-PD-1 and anti-CTLA4 mediated G2-3 colitis consists in intravenous (IV) glucocorticosteroids $(1-2 \mathrm{mg} / \mathrm{kg}$ every 24 hours). If symptoms do not improve within 5 days of IV corticoids administration, $5 \mathrm{mg} / \mathrm{Kg}$ doses of the anti-TNF antibody Infliximab should be started $[5,10]$. As there is no published data on management of anti-LAG3 related colitis, we applied to our patient the treatment algorithms for anti-PD-1 and anti-CTLA4 related colitis.

In our case, as the toxicity was secondary to experimental drugs under clinical trial an experienced gastroenterologist evaluation was required to optimize medical management according to immune 
related AEs and chronic inflammatory bowel disease protocols. This case emphasizes the fact that corticosteroids are nowadays the mainstream treatment for any immunotherapy related colitis and is an evidence of efficacy in the context of anti-LAG3 treatment.

Corticosteroids doses were slowly tapered for almost two months at the outpatient evaluation and no infliximab was required as prompt recovery occurred. As in the case of immune related hepatitis, enterocolitis may persist. Hence, prolonged or repeated corticosteroid tapers (minimum of 3 weeks suggested) and/or additional immunosuppression could be required $[5,10]$.

Clinical trials are required to improve the outcomes of gastrointestinal toxicities in patients treated with immunotherapy. New approaches are needed to dilucidate the pathophysiology of gastrointestinal immune adverse effects.

As new immunotherapies are being implemented, new multidisciplinary protocols should carry into effect, registering new data in protocols which involves training and expertise from other medical departments.

The case of our patient highlights the importance of prompt identification of immune-related adverse effects by both physicians and patients, and their management. Differential diagnosis from other plausible causes is mandatory during this process in order to avoid detrimental effects from treatment. Finally, prompt instauration of immunosuppressant treatment is the last step to correctly diagnose and manage immunotherapy related symptoms.

To our concern this is the first reported case of enterocolitis in a patient treated with a combination of anti-PD-1 anti-LAG3 therapy.

\section{References}

1. Hodi FS, O’Day SJ, McDermott FD, Weber RW, Sosman AJ, et al. (2010) Improved survival with Ipilimumab in patients with metastatic melanoma. N Engl J Med 363(8): 211-223.

2. Robert C, Long GV, Brady B, Dutriaux C, Maio M, et al. (2015) Nivolumab in previously untreated malanoma without BRAF mutation. $\mathrm{N}$ Engl J Med 372(4): 320-330.

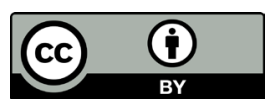

This work is licensed under Creative Commons Attribution 4.0 License DOI: 10.19080/JTMP.2018.03.555602
3. Larkin J, Chiarion-Sileni V, Gonzalez R, Grob JJ, Cowey L, et al. (2015) Combined nivolumab and ipilimumab or monotherapy in untreated melanoma. N Engl J Med 373(13): 1270-1271.

4. Robert C, Karaszewska B, Schachter J, Rutkowski P, Mackiewicz A, et al. (2015) Improved overall survival in melanoma with combined dabrafenib and trametinib. N Engl J Med 372(1): 30-39.

5. Boutros C, Tarhini A, Routier E, Lambotte O, Ladurie FL, et al. (2016) Safety profiles of anti-CTLA-4 and anti-PD-1 antibodies alone and in combination. Nat Rev Clin Oncol 13(8): 473-486.

6. Hodi SF, Kluger HM, Sznol M, Carvajal R, Lawrence, et al. (2016) Durable, long-term survival in previously treated patients with advanced melanoma who received nivolumab monotherapy in a phase I trial. Cancer Research 76(14): CT001.

7. Demeure CE, Wolfers J, Martin-Garcia N, Gaulard P, Triebel F (2001) T Lymphocytes infiltrating various tumour types express the MHC class II ligand lymphocyte activation gene-3 (LAG-3): role of LAG-3/MHC class II interactions in cell-cell contacts. Eur J Cancer 37(13): 17091718.

8. Workman CJ, Cauley LS, Kim IJ, Blackman MA, Woodland DL, et al. (2004) Lymphocyte activation gene-3 (CD223) regulates the size of the expanding $\mathrm{T}$ cell population following antigen activation in vivo. J Immunol 172(9): 5450-5455.

9. Verschuren EC, van den Eertwegh AJ, Wonders J, Slangen RM, van Delft F, et al. (2016) Clinical, endoscopic, and histologic characteristics of ipilimumab-associated colitis. Clin Gastroenterol Hepatol 14(6): 836842 .

10. Naidoo J, Page DB, Li BT, Connell LC, Schindler K, et al. (2015) Toxicities of the anti-PD-1 and anti-PD-L1 immune checkpoint antibodies. Ann Oncol 26(12): 2375-2391.

11. Woo SR, Turnis ME, Goldberg MV, Bankoti J, Selby M, et al. (2012) Immune inhibitory molecules LAG-3 and PD-1 synergistically regulate T-cell function to promote tumoral immune escape. Cancer Res 72(4): 917-927.

12. Jain A, Lipson EJ, Sharfman WH, Brant SR, Lazarev MG (2017) Colonic ulcerations may predict steroid-refractory course in patients with ipilimumab-mediated enterocolitis. World J Gastroenterol 23(11): 2023-2028.

\section{Your next submission with Juniper Publishers will reach you the below assets}

- Quality Editorial service

- Swift Peer Review

- Reprints availability

- E-prints Service

- Manuscript Podcast for convenient understanding

- Global attainment for your research

- Manuscript accessibility in different formats

( Pdf, E-pub, Full Text, Audio)

- Unceasing customer service

Track the below URL for one-step submission https://juniperpublishers.com/online-submission.php 